\title{
Improved Motion Correction in fMRI by Joint Mapping of Slices into an Anatomical Volume
}

\author{
Hyunjin Park, Charles R. Meyer, and Boklye Kim \\ Department of Radiology, University of Michigan Medical School, MI 48109, USA \\ \{hyunjinp, cmeyer, boklyek\}@umich.edu \\ http: / /www.med.umich.edu/dipl
}

\begin{abstract}
Motion correction in fMRI time series is essential for accurate statistical analyses. Typically motion correction is applied in a rigid fashion between time series volumes. Such corrections assume no relative motion between slices. An improved motion correction scheme, map-slice-to-volume (MSV), was developed previously using mutual information (MI) to register individual fMRI slices onto an anatomical volume to account for inter-slice motion [6]. As each slice's orientation represents a statistically independent sampling of the patient's motion at multiple intervals throughout the acquisition, a smoothed estimate of the patient's trajectory in time can be computed by jointly estimating each slice's orientation while minimizing the implied acceleration of the patient's head subject to applying a priori, slice-related weights based on known registration reliabilities. The results of this joint mapping of slices into a volume (JMSV) show further substantial improvement in slice registration and subsequent motion estimation.
\end{abstract}

\section{Introduction}

Subject head motion is a major obstacle in accurate measurements of voxel intensity changes in fMRI. A widely used method is to register each EPI (echo planar imaging) volume (i.e., a stack EPI slices) in an fMRI time series onto a reference EPI volume volumetrically $[4,5]$. This method assumes that there is no inter-slice motion, i.e. individual slices within a volume do not move independently in the subject's frame of reference. This assumption is valid only when there is very little motion as might occur with the subject's head wedged into the head coil with MRI-compatible packing materials during finger tapping. However, in the presence of frank motion as might occur in a verbalized speech individually acquired slices are no longer parallel in the subject's frame of reference. During the acquisitions of sequential slices in an interleaved acquisition, sequential slices may have significantly different motion trajectories relative to the geometric reference frame of the subject. Previously a new motion correction scheme, MSV (Map Slice to Volume), that can account for inter-slice relative motion was developed and demonstrated an improved capability in correcting image shifts due to the rigid head motion [6]. It allows individual slices within a volume to be mapped onto the subject's anatomic volume reference before statistical testing for activation is performed. Use of the MSV method shows improved sensitivity and specificity in locating activated regions compared to other widely used, parallel slice-stack based methods, e.g. statistical parametric mapping (SPM).

The MSV method uses mutual information as the objective function in the automatic image registration process. For the most part, the estimated position parameters 
from the middle slices of the brain are accurate since there is sufficient information from a large region of support to drive the registration. The reliability of the estimated position parameters for the end cap slices, however, is reduced due to lower information content created by smaller regions of support. Our goal is to improve not only the accuracy of the registration of the end cap slices but the accuracy of the middle slices as well by imposing the condition that the motion trajectory of the subject's head as measured by the resulting time series of slice orientations is acceleration limited, i.e. smooth. In MSV we estimated the position of each slice independently of others. Here we present a method of jointly estimating the position of multiple slices in the time series using MI subject to simultaneously minimizing the associated acceleration in the motion trajectory of the subject. This new motion correction method is referred as the Joint Mapping of Slices into Volume (JMSV) method.

\section{Motion Correction Methods}

The goal of an fMRI experiment is to localize regions of activation in response to certain stimuli. Typically a time series of low resolution fMRI data are acquired using GRE (gradient recalled echo) -EPI sequence at the rate of 2 to 3 seconds per volume. The image matrix size is typically $128 \times 128$ or $64 \times 64$. In a block design, alternating phases of activation and rest are repeated. A high resolution anatomical reference volume is acquired following the fMRI session. Statistical difference in image intensities between the activation and rest images is used to determine whether the region is active or not. The statistical analysis assumes that the EPI scans are all aligned to a chosen reference scan. Thus, at a given location in the reference scan, it is comparing MR signals coming from the same location over different EPI volumes. An accurate motion correction that guarantees consistent voxel location is essential for accurate statistical analysis. Whereas the commonly used SPM method based on a volumetric rigid registration of EPI volumes works only for cases of limited motion, MSV can correct more complex motion by allowing slices within a volume to move independently and provide an effective localization capability [6]. Here we improve on the results of the MSV method by jointly registering multiple slices onto the subject's anatomical volume.

\subsection{Motion Correction by Map Slice to Volume (MSV)}

The registration process in MSV is inter-modality, since it registers the $\mathrm{T}_{2}{ }^{*}$-weighted EPI slices with the $T_{1}$-weighted anatomical scan. MSV uses mutual information (MI) as the similarity measure and 6 DOF rigid transform for the geometric model. MI is an effective similarity measure for inter-modality registrations $[2,3]$. The MI used here is the classical Shannon MI where all PDFs, both marginal and joint PDFs, are estimated by a histogram with an appropriately chosen number of bins [2]. The optimizer is the Nelder-Mead simplex optimizer. The following equation (1) is the formulation of the MSV method. The motion correction capabilities of MSV provide improved sensitivity and specificity in localizing activated regions compared to the widely used volume registration methods where the volume is assumed to consist of a set of parallel stack of slices. 
$\hat{\theta}_{i}=\underset{\theta}{\arg \max } M I\left(S_{i}(\theta), V_{r e f}\right) \quad i=0, . ., N M-1$

$\theta=\left(t_{x}, t_{y}, t_{z}, \operatorname{rot}_{x}, \operatorname{rot}_{y}\right.$, rot $\left._{z}\right)$; rigid motion param.

$\hat{\theta}_{i}$; estimated motion param. for i-th EPI slice

$S_{i}$; i-th EPI slice $V_{\text {ref }}$; anatomical volume

N;No. of EPI scans M; No. of slices per EPI scan

\subsection{Joint Mapping of Slices into Volume (JMSV) Method}

Sometimes the estimated motion parameters for sequentially acquired slices in a volume using the MSV method can be noisy; at one slice the estimated rotation about $\mathrm{z}$ axis could be at 2 degrees and then change to -5 degrees at the subsequently acquired slice. This kind of abrupt change in the motion parameters implies very unlikely motion, since it requires a sudden, large acceleration. This abnormality in the motion parameters typically occurs either at the top or the bottom slices of the EPI head scan. The MSV method provides accurate estimations in motion parameters for slices with sufficient information, i.e., enough detail and no severe geometric distortion. Towards the top slice of the brain, however, slices contain less information (i.e. less textured area and more background) than the slices from the mid-brain region and may not have enough information for the stable estimation of slice position. In the regions near the bottom of the head, slices contain significant geometric distortion, especially where air/tissue interface occurs [7], and this in turn negatively affects the reliability of the motion parameter estimates. Here we implement a joint estimation of the registration of slices while penalizing the implied acceleration. The formulation of the new motion correction method, referred as Jointly Mapping Slices into a Volume (JMSV) method, follows.

$$
\begin{aligned}
& \hat{\theta}=\underset{\theta}{\arg \max }\left\{\sum_{i=0}^{K-1} w_{i} M I\left(S_{i}\left(\theta_{i}\right), V_{\text {ref }}\right)-\beta R(\theta)\right\} \\
& =\underset{\theta}{\arg \max }\left\{\sum_{i=0}^{K-1}\left(w_{i} M I\left(S_{i}\left(\theta_{i}\right), V_{\text {ref }}\right)-\beta\left(\left(\theta_{i}-\theta_{i-1}\right)-\left(\theta_{i+1}-\theta_{i}\right)\right)^{2}\right)\right\} \\
& \theta_{i} ; \text { motion param. for i-th EPI slice } \\
& \theta=\left(\theta_{0}, . ., \theta_{K-1}\right) ; \text { collection of motion param. over K slices } \\
& \hat{\theta} ; \text { estimated motion param. for K slices } \\
& S_{i} ; \text { i-th EPI slice } V_{\text {ref }} ; \text { anatomical volume } \\
& w_{i} ; \text { weight associated with MI value of the i-th slice } \\
& R(\theta) ; \text { roughness penalty } \beta \text {; weight for roughness penalty }
\end{aligned}
$$


Motion parameters for $\mathrm{K}$ slices are jointly estimated by maximizing the objective function in equation (2). The number $\mathrm{K}$, the number of jointly registered slices, may be as small as 3 or as large as the number of slices in the entire time series. Smoothness of the motion parameters is implemented through a roughness penalty term $R(\theta)$ in the objective function. We have chosen a discrete $2^{\text {nd }}$ order difference roughness penalty. This roughness penalty encourages the motion parameters of sequentially acquired slices to have constant angular and translational velocities. As noted before, some slices may lead to less reliable motion parameter estimations, thus the MI values of those slices should be weighted less than the MI values from more reliable slices in the objective function. For example, we know a priori that the top slice and the bottom slice of the brain should be weighted less than the slices in between. In this paper, we used both a flat weighting that does not account for the less reliable slices and a linear weighting that starts at 1 for the middle slice and decreases to 0.05 for both the top slice and the bottom slice. The linear weighting has a triangular waveform when plotted with respect to the location of the slice. Additional computational effort is required to perform the JMSV method since it is jointly estimating the motion parameters for multiple slices rather than estimating the motion parameters for one slice at a time as in MSV. Also it is worthy to note that in case of no roughness penalty (i.e. $\beta=0$ ) and a flat weighting for MI values (i.e. $\mathrm{w}_{\mathrm{i}}=1$ ), the JMSV method reduces to the MSV method.

\section{Experiments}

A data set for typical fMRI time series were acquired using GRE-EPI sequence on a GE Signa system operating at field strength of 1.5 Tesla. The acquisition parameters were: $6 \mathrm{~mm}$ slice thickness with no gaps, $\mathrm{FOV}=24 \times 24 \mathrm{~cm}$ in $128 \times 128$ matrix. $\mathrm{A} \mathrm{T}_{1^{-}}$ weighted high resolution anatomical reference volume was acquired following the fMRI session using 3D spoiled GRASS (SPGR) sequence where the slice thickness = $1.5 \mathrm{~mm}$, and the $\mathrm{FOV}=24 \times 24 \mathrm{~cm}$ in a $256 \times 256$ matrix. For an experiment to test the algorithm, an EPI volume, that was believed to be almost motionless, acquired with no activation, i.e. a dry run, was used. Figure 1 shows a corresponding pair of slices from the EPI dry run and $\mathrm{T}_{1}$ weighted anatomical volume.

We have applied a series of synthetic rigid motion to individual slices of the dry run to create simulated EPI volumes where the ground truth for the motion is established. The series of synthetic motion was applied in the order of the slice acquisition, not the order of physical stacking of the slices as in figure 2. The synthetic motion was only applied as in-plane rotation about $\mathrm{z}$ axis; the direction of inferior/superior. The applied motion is $\operatorname{rot}\{z(j)\}=3 \sin (\mathrm{j} / 4.7746)$, where $\mathrm{j}$ is the index of the slice acquisition order and $\operatorname{rot}\{z\}$ is the value of rotation about $z$ axis. Two synthetic EPI volumes were created by letting $\mathrm{j}$ vary from 0 to 31 . Both the MSV and JMSV methods have been applied to recover the motion. For the JMSV method, the flat and triangular weighting, as described earlier, were applied in separate runs and compared. Root mean squared (RMS) errors between the estimated and true motions are reported in Table 1. As shown in table 1, the error is the largest for MSV; the error is reduced 
using JMSV with uniform weighting, and finally the error is smallest using JMSV with triangular weighting. Figure 3 shows the recovered motion parameters for all the motion correction methods. Typically EPI slices are acquired in an interleaving fashion as in Figure 2. Thus the top two slices occur at the slice acquisition indices 0 and 8, and the bottom two slices at 7 and 15. For the second EPI volume of 16 slices, the top two slices occur at the acquisition indices 16 and 24 and the bottom two occur at 23 and 31. In Figure 3, MSV shows abrupt changes on the slice acquisition indices, 0 , 8,15 , and 24 , all of which belong to either the top two or the bottom two slices. Improvement is noticeable just by exploiting the correlation of the motion parameters. The RMS error decreased from 1.06 to 0.59 by going from MSV to JMSV with the flat weights. Additional improvement was achieved by incorporating a smart weighting; the RMS error decreased to 0.47 for the JMSV method with the triangular weights.
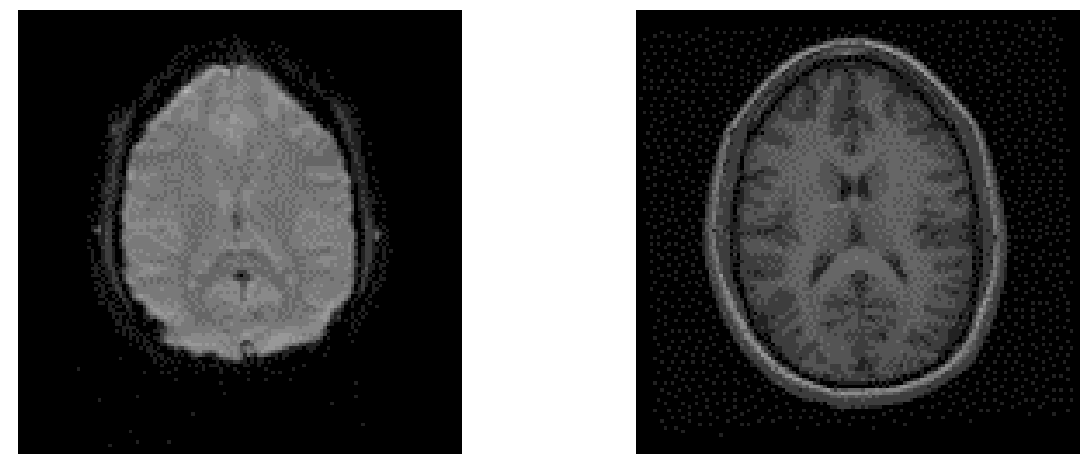

Fig. 1. A corresponding pair of slices from the EPI and anatomical data sets selected from the $3 \mathrm{D}$ data sets. The left is the EPI slice and the right is the anatomical $\mathrm{T}_{1}$ slice. The slices are located in about mid brain.

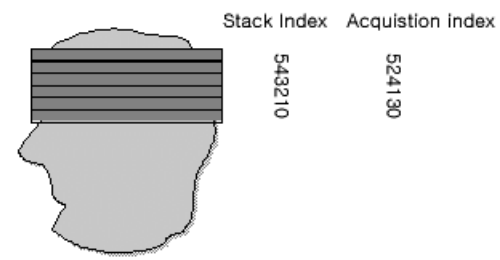

Fig. 2. Comparison of slice acquisition index and slice stacking index of an EPI scan.

\section{Summary}

Our previous work with MSV demonstrated an effective rigid motion correction by allowing individual slices to move independently as each slice is subject to different motion [6]. Improvement in registration noise reduction is desirable since the regis- 
tration accuracies were less reliable for slices from the extreme locations of the brain. In this paper, we propose to improve our MSV method by minimizing the implied acceleration for sequentially acquired slices and adopting a slice-based weighting for



Fig. 3. Recovered motion parameters for different motion correction methods. The true motion parameters are shown in solid red. The estimated motion parameters from MSV, JMSV with uniform weights, and JMSV with triangular weights are in dotted green, dash dotted blue, and dashed yellow respectively. For the MSV method abrupt changes in motion parameters can be observed at the acquisition index $0,8,15$, and 24 (i.e. marked with arrows). These abrupt changes are smoothed using the JMSV method. There is a substantial improvement by using JMSV instead of MSV. Within the JMSV method, imposing a slice-based weighting related to the variance of the MSV estimate (i.e. triangular weights) further reduces the error as shown in table 1, but this improvement is less noticeable than going from MSV to JMSV.

Table 1. Root Mean Squre (RMS) error associated with different motion correction methods.

\begin{tabular}{|c|c|}
\hline Methods & RMS Error [degrees] \\
\hline MSV & 1.06 \\
\hline JMSV with uniform weights & 0.59 \\
\hline JMSV with triangular weights & 0.47 \\
\hline
\end{tabular}

the similarity measure. Although motion correction methods (i.e. MSV, JMSV with uniform and triangular weights) have been performed on a simulated data set, we believe the error reduction between the methods proves to be meaningful. Future work will include multiple runs of the motion correction methods to establish statistical comparisons. Also, we used triangular weighting to account for a priori knowledge of the reliability of the estimated motion parameters. This simple weighting can 
be further improved by using appropriately estimated variance measures for the registration accuracy of each slice position across subjects. While the JMSV method requires additional computation to implement the joint registration of multiple slices, we believe that the accuracy gained by improved motion correction warrants such costs.

\section{References}

1. Jenkinson, M., Smith, S.: The role of registration in functional magnetic resonance imaging. In: Hajnal, J. V., Hill, D. L. G., Hawkes, D. J.(eds.): Medical image registration. CRC press, 2001 183-198.

2. Hill, D. L. G., Batchelor, P. G., Holden, M., Hawkes, D. J.: Medical image registration. Physics in medicine and biology 46 (2001) r1-r45.

3. Meyer, C. R., Boes, J. L., Kim, B., Bland, P. H.: Demonstration of accuracy and clinical versatility of mutual information for automatic multimodality image fusion using affine and thin plate spline warped geometric deformations. Medical image analysis 3 (1997) 195-206.

4. Friston, K. J., Williams, S. R., Howard, R., Frackowiak, R. S. J., Turner, R.: Movement related effects in fMRI time-series. Magnetic resonance in medicine 35 (1996) 346-355.

5. Hajnal, J. V., Myers, R., Oatridge, A., Schwieso, J. E., Young, I. R., Bydder, G. M.: Artefacts due to stimulus correlated motion in functional imaging of the brain. Magnetic resonance in medicine 31 (1994) 283-291.

6. Kim, B., Boes, J. L., Bland, P. H., Chenervert, T. L., Meyer, C. R.: Motion correction in fMRI via registration of individual slices into an anatomical volume. Magnetic resonance in medicine 41 (1999) 964-972.

7. Jezzard, B., Balaban, R. S.: Correction for geometric distortion in echo planar images from B0 field variations. Magnetic resonance in medicine 34 (1995) 65-73. 